\title{
Endothelial Dysfunction, Atherosclerosis, and Increase of von Willebrand Factor and Factor VIII: A Randomized Controlled Trial in Swine
}

\author{
Moniek P. M. de Maat ${ }^{1}$ Daphne Merkus $2,5,6[$ \\ ${ }^{1}$ Department of Hematology, Erasmus Medical Center, University \\ Medical Center Rotterdam, Rotterdam, The Netherlands \\ 2 Division of Experimental Cardiology, Department of Cardiology, \\ Thoraxcenter, Erasmus Medical Center, University Medical Center \\ Rotterdam, Rotterdam, The Netherlands \\ ${ }^{3}$ Turku PET Centre, University of Turku and Turku University Hospital, \\ Turku, Finland \\ ${ }^{4}$ Rydberg Laboratory of Applied Sciences, University of Halmstad, \\ Halmstad, Sweden \\ ${ }^{5}$ Walter Brendel Center of Experimental Medicine (WBex), LMU \\ Munich, Munich, Germany \\ ${ }^{6}$ German Center for Cardiovascular Research (DZHK), Partner Site \\ Munich, Munich Heart Alliance (MHA), Munich, Germany
}

Ferdows Atiq ${ }^{1, *(i)}$ Jens van de Wouw ${ }^{2, *(1)}$ Oana Sorop ${ }^{2(0)}$ Ilkka Heinonen ${ }^{2,3,4(0)}$

Dirk J. Duncker ${ }^{2}$ Frank W. G. Leebeek ${ }^{10}$ Address for correspondence Frank W. G. Leebeek, MD, PhD,
Department of Hematology, Erasmus University Medical Center, PO
Box 2040, 3000 CA Rotterdam, The Netherlands
(e-mail: F.leebeek@erasmusmc.nl).

Abstract



Keywords
- von Willebrand factor
- von Willebrand
disease
- diabetes mellitus
- endothelial
dysfunction
- atherosclerosis
- cardiovascular
disease
- swine

It is well known that high von Willebrand factor (VWF) and factor VIII (FVIII) levels are associated with an increased risk of cardiovascular disease. It is still debated whether VWF and FVIII are biomarkers of endothelial dysfunction and atherosclerosis or whether they have a direct causative role. Therefore, we aimed to unravel the pathophysiological pathways of increased VWF and FVIII levels associated with cardiovascular risk factors. First, we performed a randomized controlled trial in 34 Göttingen miniswine. Diabetes mellitus (DM) was induced with streptozotocin and hypercholesterolemia $(\mathrm{HC})$ via a high-fat diet in 18 swine (DM + HC), while 16 healthy swine served as controls. After 5 months of follow-up, FVIII activity (FVIII:C) was significantly higher in $\mathrm{DM}+\mathrm{HC}$ swine $(5.85 \mathrm{IU} / \mathrm{mL}$ [5.00-6.81]) compared with controls (4.57 [3.76-5.40], $p=0.010$ ), whereas VWF antigen (VWF:Ag) was similar (respectively 0.34 $\mathrm{IU} / \mathrm{mL}[0.28-0.39]$ vs. 0.34 [0.31-0.38], $p=0.644$ ). $\mathrm{DM}+\mathrm{HC}$ swine had no endothelial dysfunction or atherosclerosis during this short-term follow-up. Subsequently, we performed a long-term (15 months) longitudinal cohort study in 10 Landrace-Yorkshire swine, in five of which $\mathrm{HC}$ and in five combined DM + HC were induced. VWF:Ag was higher at 15 months compared with 9 months in $\mathrm{HC}(0.37$ [0.32-0.42] vs. 0.27 [0.23-0.40], $p=0.042)$ and $\mathrm{DM}+\mathrm{HC}(0.33$ [0.32-0.37] vs. 0.25 [0.24-0.33], $p=0.042)$. Both long-term groups had endothelial dysfunction compared with controls and atherosclerosis after 15 months. In conclusion, short-term hyperglycemia and dyslipidemia increase FVIII, independent of VWF. Long-term DM and HC increase VWF via endothelial dysfunction and atherosclerosis. Therefore, VWF seems to be a biomarker for advanced cardiovascular disease.

These authors contributed equally to this work.

received

August 14, 2020

accepted after revision

November 9, 2020

published online

January 14, 2021 (c) 2021. Thieme. All rights reserved. Georg Thieme Verlag KG,

Rüdigerstraße 14,

70469 Stuttgart, Germany
DOI https://doi.org/

10.1055/s-0040-1722185.

ISSN 0340-6245. 


\section{Introduction}

von Willebrand factor (VWF), which is mainly produced in endothelial cells, is a prohemostatic protein with an important role in hemostasis. ${ }^{1,2}$ The primary function of VWF is to mediate platelet adhesion and aggregation. ${ }^{1,2}$ VWF is also a carrier protein for factor VIII (FVIII), and thereby prevents FVIII degradation. Consequently, plasma levels of VWF and FVIII are strongly correlated., ${ }^{1,2}$

It is known from large-population studies that high VWF levels are associated with an increased risk of cardiovascular disease, such as acute myocardial infarction and ischemic stroke, worse outcome of cardiovascular disease, and cardiovascular mortality. ${ }^{3-7}$ VWFand FVIII levels have been shown to be influenced by genetic factors, age, blood group, physical stress, and several other environmental factors. ${ }^{8-10}$ For decades it was thought that aging directly leads to an increase in VWF and FVIII levels. ${ }^{8,9,11,12}$ However, recently we have shown that incidence of comorbidities such as diabetes mellitus (DM), hypertension, cancer, and thyroid dysfunction at an older age may explain the association between aging and increased VWF and FVIII levels. ${ }^{10}$ Nevertheless, due to the cross-sectional design of that study, it could not be determined whether there was a causal relation between comorbidities and the increase in VWF and FVIII levels. Moreover, we could not investigate whether comorbidities directly lead to an increase in VWF and FVIII levels, or whether this occurs secondary to endothelial dysfunction or atherosclerosis. Indeed, previous studies found that VWF levels were associated with carotid artery and aortic arch calcification, suggesting that increased VWF levels may be the result of endothelial dysfunction or atherosclerosis. 5,6

Since the association between VWF and cardiovascular disease and mortality is still not fully understood, it is important to gain more insight into the pathophysiological pathways linking cardiovascular risk factors with the increase in VWF and FVIII levels. ${ }^{13-15}$ On one hand, increased VWF and FVIII levels may be strong, independent risk factors for cardiovascular disease and mortality. ${ }^{14,15}$ On the other hand, the association between increased VWF and FVIII levels and cardiovascular disease and mortality may be partly confounded by comorbidities, such as DM and hypercholesterolemia (HC), which are also well-established risk factors for cardiovascular disease and mortality. ${ }^{13}$ Moreover, it is still debated whether increased VWF and FVIII levels lead to endothelial dysfunction and atherosclerosis or vice versa. ${ }^{13}$ The large population studies on which most of our current knowledge about the association between VWF and cardiovascular disease and mortality are based could not determine the pathophysiological pathways underlying the association between VWF, endothelial dysfunction, atherosclerosis, and cardiovascular disease and outcomes. Knowledge about these pathways may contribute to a better understanding of previous studies and to establish whether VWF is merely a biomarker for endothelial dysfunction and atherosclerosis or whether it has a direct causative role, making it suitable as a drug target for prevention or treatment of cardiovascular disease. ${ }^{13}$

To address these questions, we first investigated the pathophysiological pathways of the comorbidity-related in- crease of VWF and FVIII levels in a randomized controlled trial (RCT) design in swine with short-term (5 months) $\mathrm{DM}+\mathrm{HC}$, to study the direct effects of these comorbidities on VWF and FVIII levels, at a time when endothelial dysfunction and atherosclerosis were still absent. Subsequently, we studied VWF levels in swine exposed to longer term (15 months) $\mathrm{DM}+\mathrm{HC}$ at a time when endothelial dysfunction and atherosclerosis were present.

\section{Methods}

We studied two cohorts of swine. In the first cohort Göttingen miniswine were subjected to 5 months of $\mathrm{DM}+\mathrm{HC}$, while in the second cohort Landrace-Yorkshire farm swine were subjected to 15 months of $\mathrm{DM}+\mathrm{HC}$ or $\mathrm{HC}$ alone. All experiments were conducted in a time period of 20 months, animals were ordered in groups of eight or nine animals, and each group was evenly randomized to prevent selection bias and minimize the possibility of time-dependent confounding. All experiments were performed in accordance with the National Institutes of Health Guide for the Care and Use of Laboratory Animals (eighth edition, National Research Council, Washington, DC, The National Academies Press, 2011) and were approved by the Animal Care Committee at Erasmus Medical Center.

\section{Short-Term Cohort}

Induction of Metabolic Derangement in Swine

A total of 34 adult male Göttingen miniswine (Ellegaard Göttingen Miniswine A/S, Dalmose, Denmark) were enrolled in the first part of the study. Swine were randomized into an experimental group in which DM and HC were induced, and a control group which continued on normal swine chow. DM was induced with intravenous injections of streptozotocin ( $25 \mathrm{mg} / \mathrm{kg} / \mathrm{d}$ ), over 3 days (total dose: $75 \mathrm{mg} / \mathrm{kg}$ ) as previously described. ${ }^{16}$ One week later an isocaloric high-fat and highsugar diet $(25 \%$ of saturated fats, $1 \%$ of cholesterol, $10 \%$ sucrose, and $15 \%$ fructose) was gradually introduced in these swine. ${ }^{16}$ Swine were group-housed with a separate individual access to food for 1 hour/meal, twice daily for the entire study duration, and ad libitum access to water. All swine were followed up for 5 months, to study the short-term effect of DM + HC on VWF and FVIII levels.

Of note, after 3 months of follow-up, both control and $\mathrm{DM}+\mathrm{HC}$ groups were further subdivided into a subgroup exposed to exercise training and a sedentary subgroup. Since exercise training did not influence the results and conclusions of the current study, we pooled the exercised and sedentary swine, and present the results for the pooled $\mathrm{DM}+\mathrm{HC}$ and pooled control group. The influence of exercise on VWF and FVIII levels are presented in - Supplementary Table S1 (available in the online version).

\section{Plasma Metabolic, VWF, and FVIII Laboratory Measurements}

Venous blood samples were obtained from lightly sedated animals by insertion of a 20 Gauge Venflon into an ear vein. 
Blood was collected in a heparin-coated collecting tube at fasting state at baseline, 3-month, and 5-month (termination) time points, and analyzed for glucose, triglyceride, and cholesterol using standardized human protocols at the clinical chemical laboratory of the Erasmus Medical Center, Rotterdam. Venous blood samples were also collected in collecting tubes containing 3.2\% trisodium citrate, centrifuged, and stored at $-80^{\circ} \mathrm{C}$ for analyses of VWF and FVIII. VWF antigen (VWF:Ag) was measured with an in-house enzyme-linked immunosorbent assay using polyclonal rabbit anti-human VWF antibodies (DakoCytomation, Glostrup, Denmark). It has previously been shown that human VWF assays can be used to measure VWF in porcine plasma. ${ }^{17} \mathrm{FVIII}$ activity (FVIII:C) was measured using a one-stage clotting assay on the Sysmex CS-5100 (Siemens).

Hemodynamic Assessments, Coronary Angiography, and Cardiac Tissue Sampling and Analyses

After 5 months (at termination), animals were sedated with zoletil (Tiletamine/Zolazepam; $5 \mathrm{mg} / \mathrm{kg}$ intramuscular), rompun (xylazine; $2.25 \mathrm{mg} / \mathrm{kg}$ intramuscular), and atropine ( $2 \mathrm{~mL}$ intramuscular), anesthetized with pentobarbital (20 $\mathrm{mg} / \mathrm{kg}$ intravenous) and artificially ventilated. Thereafter, a 9F sheath was placed in the left carotid artery and a standard clinical guiding catheter (JL3.5, Boston Scientific) was advanced through the carotid artery to the coronary ostium for angiography of the coronary arteries using iodixanol as a contrast agent (Visipaque, GE Healthcare BV, Eindhoven, The Netherlands). Following thoracotomy, hearts were arrested, immediately excised ,and quickly washed in cold saline solution and then the apex was placed in ice-cold, oxygenated Krebs bicarbonate buffer solution. Subsequently, epicardial small coronary arteries $(\sim 300 \mu \mathrm{m}$ diameter $)$ were isolated of both groups for functional experiments. The large coronary arteries were fixed in $4 \%$ buffered formaldehyde, embedded in paraffin, and stained with resorcin-fuchsin to assess atherosclerosis development in both groups.

\section{Microvascular Function In Vitro}

The isolated segments were studied in vitro in a Mulvany wire myograph (DMT, Aarhus, Denmark) as previously described. ${ }^{18}$ In short, after a 30 -minute stabilization period, vessels were normalized to $90 \%$ of the estimated diameter at $100 \mathrm{mmHg}$ effective transmural pressure. Thereafter, the vascular segments were depolarized by $100 \mathrm{mM} \mathrm{KCl}$ (SigmaAldrich, Zwijndrecht, The Netherlands) to determine the maximal contractile response of the small-artery segments. Subsequently, vasodilator or vasoconstrictor responses to various substances (all bought from Sigma-Aldrich) were acquired using separate, but in vivo adjacently positioned, segments. Endothelium-dependent vasodilation was studied by measurement of vasodilation in response to bradykinin (BK $10^{-8} \mathrm{M}$ ) following preconstriction by the thromboxane A2 analog U46619 $\left(10^{-6} \mathrm{M}\right)$. Endothelium-independent vasodilation was studied in separate small-artery segments by measuring the vasodilatory response to the exogenous NOdonor S-nitroso-N-acetyl-D,L-penicillamine (SNAP $10^{-7} \mathrm{M}$ ). In additional small-artery segments, vasoconstriction to endothelin-1 (ET-1 $10^{-8} \mathrm{M}$ ) was measured. Previous research studies from our group and others have demonstrated a group size of five to eight swine to be sufficient to detect biological relevant differences in coronary microvascular function ( $80 \%$ confidence, two-tailed test, $\alpha=0.05){ }^{18-23}$

\section{Long-Term Longitudinal Cohort}

The longitudinal cohort was described in detail previously. ${ }^{18}$ Briefly, 10 Landrace-Yorkshire swine were included in the study at 3 months of age. In five swine DM was induced with a single intravenous injection of streptozotocin (140-180 $\mathrm{mg} / \mathrm{kg}$ ). In the DM swine and the remaining five swine without DM, HC was induced with a high-fat and high-sugar diet, as described above. Food intake was monitored for each animal separately and titrated to maintain growth at approximately $1.5 \mathrm{~kg} /$ week. After 9,12 , and 15 months of exposure to $\mathrm{HC}$ or $\mathrm{DM}+\mathrm{HC}$, arterial citrate plasma samples were collected to measure VWF:Ag, and coronary angiograms were recorded, as described above. After 15 months of induction of $\mathrm{DM}+\mathrm{HC}$, animals were terminated and microvascular function and large coronary atherosclerosis development were determined, as described above. Additionally, 12 fresh slaughterhouse swine hearts were collected as controls to assess small coronary artery function.

\section{Statistical Analysis}

Continuous data are presented as median and interquartile range (IQR; in square brackets), whereas categorical data are presented as frequency and proportion (in first parentheses). Normality of data was visually assessed with histograms. Because VWF:Ag and FVIII:C were not normally distributed, nonparametric tests were used. The Mann-Whitney U test was used to analyze a difference between two independent groups, whereas the Kruskal-Wallis test was used to analyze a difference between three groups. A difference between two related groups was analyzed with the Wilcoxon signed-rank test.

The correlation between VWF and FVIII was assessed with Spearman's correlation analysis. Outcomes of this analysis are presented as Spearman's rho $(\rho)$ and $p$-value. The association between the FVIII:C/VWF:Ag ratio and cholesterol levels was calculated with linear regression analysis, since the FVIII:C/VWF:Ag ratio was normally distributed. Outcomes of linear regression analysis are presented as unstandardized $\beta$ and $p$-value.

A $p$-value below 0.05 was defined as statistically significant. Analyses were performed with SPSS Statistics Version 25.0 (IBM Corp. Armonk, New York, United States).

\section{Results}

\section{Short-Term Cohort}

Thirty-four adult male Göttingen miniswine were included in our RCT, of which 18 swine were randomized to the experimental group $(\mathrm{DM}+\mathrm{HC})$ and 16 swine to the control group. At baseline no difference was observed in age (respectively 19.0 months [16.5-19.0] vs. 18.0 months [15.5-19.0], $p=0.699$; values in median $[\mathrm{IQR}])$, body weight $(31.1 \mathrm{~kg}$ 
[25.9-36.1] vs. $27.8 \mathrm{~kg}$ [ 25.3-37.3], $p=0.931$ ), or in metabolic parameters between $\mathrm{DM}+\mathrm{HC}$ swine and control swine (-Table 1). As expected, DM + HC swine demonstrated robust hyperglycemia and dyslipidemia with triglycerides, low-density lipoprotein (LDL) cholesterol, high-density lipoprotein (HDL) cholesterol, total cholesterol, and glucose all two- to fivefold higher in DM + HC swine compared with control swine at 3 and 5 months (-Table 1). However, 5 months of exposure to hyperglycemia and dyslipidemia did not result in coronary atherosclerosis, as evidenced by the absence of atherosclerosis on the coronary angiograms and histologic preparations of coronary arteries in $\mathrm{DM}+\mathrm{HC}$ and control swine (-Fig. 1 ).

\section{Microvascular Function}

In the coronary small arteries, endothelium-dependent vasodilation to BK was similar in $\mathrm{DM}+\mathrm{HC}$ swine compared with controls at 5 months ( $p=0.497$, - Table 2). Endothelium-independent vasodilation was also similar, as the re- sponse to NO-donor SNAP was also not different between groups ( $p=0.217$ ). Vasoconstriction to ET-1 resulted also in similar responses in both groups $(p=0.739)$. Taken together, these data show the absence of endothelial dysfunction in the coronary circulation in swine up to 5 months after induction of $\mathrm{DM}+\mathrm{HC}$.

\section{VWF:Ag and FVIII:C during 5 Months of Follow-Up}

VWF:Ag was not different between $\mathrm{DM}+\mathrm{HC}$ and control swine at baseline $(0.44 \mathrm{IU} / \mathrm{mL}[0.38-0.50]$ vs. $0.40 \mathrm{IU} / \mathrm{mL}$ [0.36-0.45], $p=0.171)$. Also no difference was observed at 3 months (0.31 IU/mL [0.29-0.33] vs. $0.33 \mathrm{IU} / \mathrm{mL}$ [0.26-0.35], $p=0.191)$ or 5 months $(0.34 \mathrm{IU} / \mathrm{mL}[0.28-0.39]$ vs. 0.34 [0.31-0.38], $p=0.644$ ) of follow-up ( - Fig. 2A). Furthermore, no increase of VWF:Ag was observed between baseline and 5-month follow-up in either group ( - Fig. 2A).

FVIII:C remained unchanged over time in controls, whereas it steadily increased in DM $+\mathrm{HC}$ swine, reaching statistical significance at 5 month follow-up compared with baseline

Table 1 Characteristics of swine enrolled in the short-term RCT

\begin{tabular}{|c|c|c|c|}
\hline & $\mathrm{DM}+\mathrm{HC}(n=18)$ & Controls $(n=16)$ & $p$-Value ${ }^{a}$ \\
\hline Age at inclusion (mo) & 19.0 [16.5-19.0] & $18.0[15.5-19.0]$ & $p=0.699$ \\
\hline \multicolumn{4}{|l|}{ Weight $(\mathrm{kg})$} \\
\hline Baseline & $32.1[25.9-36.1]$ & 27.8 [25.3-37.3] & $p=0.931$ \\
\hline $3 \mathrm{mo}$ & $34.0[30.6-43.8]$ & $34.2[30.4-39.1]$ & $p=0.783$ \\
\hline $5 \mathrm{mo}$ & $37.4[29.1-42.9]$ & $35.8[34.1-39.5]$ & $p=0.904$ \\
\hline \multicolumn{4}{|l|}{ Triglycerides (mmol/L) } \\
\hline Baseline & $0.24[0.21-0.31]$ & $0.29[0.24-0.37]$ & $p=0.087$ \\
\hline $3 \mathrm{mo}$ & $0.42[0.26-1.34]$ & $0.26[0.25-0.34]$ & $p=0.031$ \\
\hline $5 \mathrm{mo}$ & $0.51[0.27-1.14]$ & $0.25[0.21-0.30]$ & $p=0.001$ \\
\hline \multicolumn{4}{|c|}{ Total cholesterol (mmol/L) } \\
\hline Baseline & $0.90[0.80-1.10]$ & $0.90[0.70-1.05]$ & $p=0.515$ \\
\hline $3 \mathrm{mo}$ & $5.35[2.45-8.35]$ & $1.15[0.83-1.38]$ & $p<0.001$ \\
\hline $5 \mathrm{mo}$ & $5.10[2.80-6.68]$ & $1.15[1.00-1.28]$ & $p<0.001$ \\
\hline \multicolumn{4}{|c|}{ HDL cholesterol (mmol/L) } \\
\hline Baseline & $0.58[0.49-0.63]$ & $0.54[0.38-0.64]$ & $p=0.490$ \\
\hline $3 \mathrm{mo}$ & $2.71[1.82-3.31]$ & $0.72[0.64-0.80]$ & $p<0.001$ \\
\hline $5 \mathrm{mo}$ & $2.61[1.75-3.20]$ & $0.68[0.61-0.97]$ & $p<0.001$ \\
\hline \multicolumn{4}{|c|}{ LDL cholesterol (mmol/L) } \\
\hline Baseline & $0.38[0.32-0.51]$ & $0.40[0.31-0.53]$ & $p=0.959$ \\
\hline $3 \mathrm{mo}$ & $2.60[0.85-5.96]$ & $0.41[0.34-0.53]$ & $p<0.001$ \\
\hline $5 \mathrm{mo}$ & $2.50[1.06-4.31]$ & $0.41[0.34-0.49]$ & $p<0.001$ \\
\hline \multicolumn{4}{|l|}{ Glucose (mmol/L) } \\
\hline Baseline & $4.8[4.2-6.0]$ & $5.7[5.3-6.6]$ & $p=0.015$ \\
\hline $3 \mathrm{mo}$ & 11.5 [7.7-17.9] & $5.7[4.5-6.4]$ & $p<0.001$ \\
\hline $5 \mathrm{mo}$ & $15.7[8.1-18.3]$ & $6.3[4.5-8.4]$ & $p<0.001$ \\
\hline
\end{tabular}

Abbreviations: DM, diabetes mellitus; HC, hypercholesterolemia; HDL, high-density lipoprotein; LDL, low-density lipoprotein; RCT, randomized controlled trial.

Note: Data are presented as median and interquartile range.

${ }^{\mathrm{a}}$ Mann-Whitney $\mathrm{U}$ test between $\mathrm{DM}+\mathrm{HC}$ and controls. 


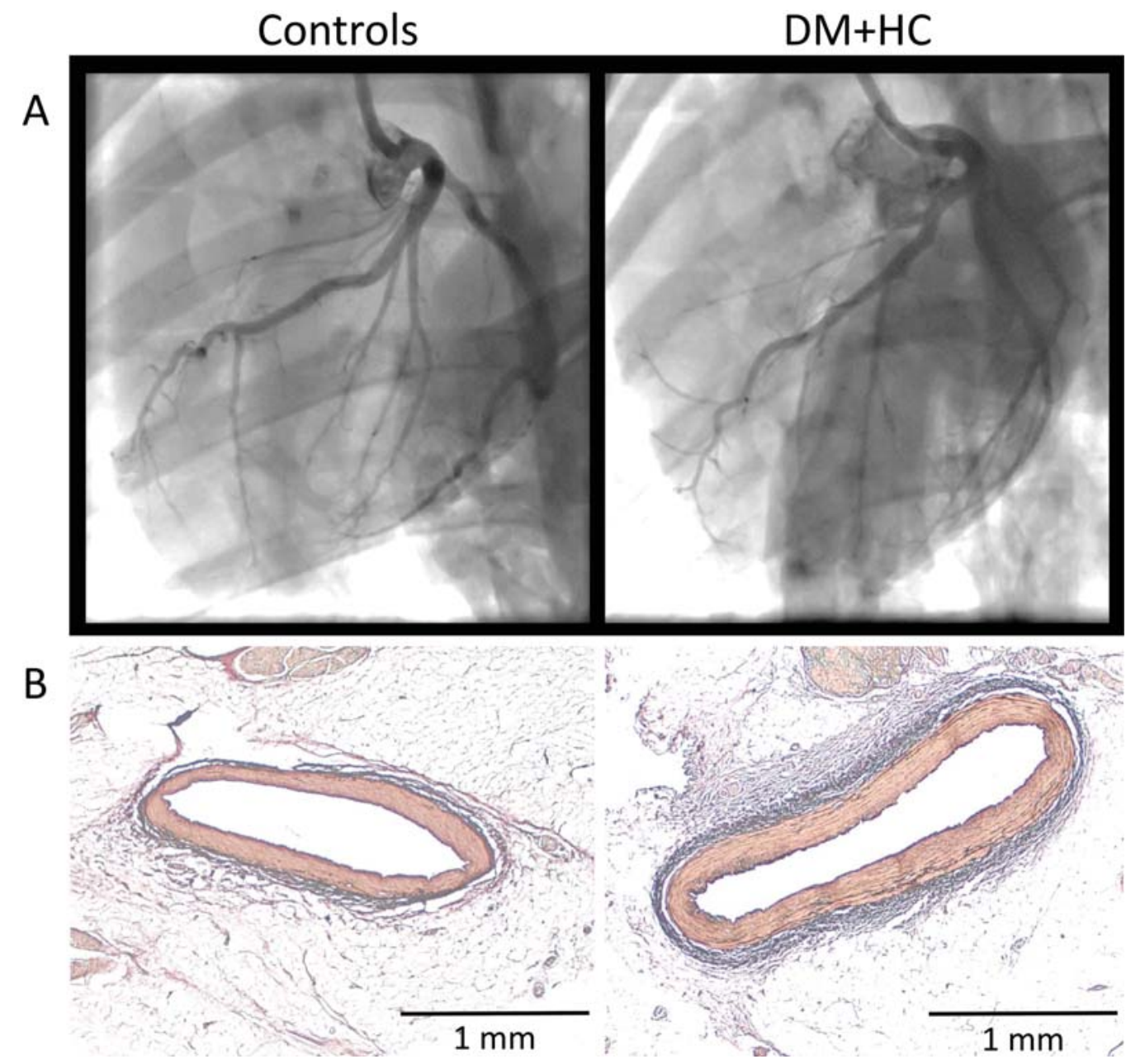

Fig. 1 No signs of atherosclerosis of coronary arteries in both $\mathrm{DM}+\mathrm{HC}$ and control swine after 5 months of follow-up. No signs of coronary atherosclerosis were observed in both groups. Typical examples of (A) coronary angiograms at a left anterior oblique $30^{\circ}$ and a cranial $0^{\circ}$ view and (B) resorcin-fuchsin stained large coronary arteries of controls and DM + HC. DM, diabetes mellitus; HC, hypercholesterolemia; VWF, von Willebrand factor.

levels ( $p=0.026$, - Fig. 2B). As a result, FVIII:C was significantly higher in $\mathrm{DM}+\mathrm{HC}$ swine compared with control swine at 5-month follow-up (respectively $5.85 \mathrm{IU} / \mathrm{mL}$ [5.00-6.81] vs. $4.57 \mathrm{IU} / \mathrm{mL}$ [3.76-5.40], $p=0.010$; - Fig. 2B).

\section{Correlation between VWF:Ag and FVIII:C}

At baseline, a strong correlation was observed between VWF: Ag and FVIII:C ( - Fig. 3A), which was statistically significant in DM + HC swine $(r=0.63, p=0.006)$. At 3-month follow-up this correlation slightly decreased in $\mathrm{DM}+\mathrm{HC}$ swine $(r=0.55, p=0.027$, - Fig. 3B), and at 5 months this correlation disappeared in DM+HC swine $(r=0.18, p=0.513)$, whereas it was strongly present in controls $(r=0.84$, $p<0.001$, - Fig. 3C). This was due to the fact that DM $+\mathrm{HC}$ swine had relatively higher FVIII:C compared with VWF:Ag at 5-month follow-up (-Fig. 3C).

Cholesterol levels were positively associated with the FVIII:C/VWF:Ag ratio at 3 and 5 months of follow-up ( - Fig. 4). The

Table 2 No difference in coronary endothelial function between DM $+\mathrm{HC}$ swine and controls in the short-term RCT

\begin{tabular}{|l|l|l|l|}
\hline & $\mathrm{DM}+\mathrm{HC}(\boldsymbol{n}=\mathbf{1 4})$ & Control $(\boldsymbol{n}=13)$ & $\boldsymbol{p}$-Value \\
\hline Bradykinin $^{\mathrm{b}}$ & $71.9 \%[49.9-90.1]$ & $53.3 \%[40.6-87.2]$ & $p=0.497$ \\
\hline SNAP $^{\mathrm{b}}$ & $34.1 \%[11.4-60.4]$ & $51.4 \%[28.5-69.1]$ & $p=0.217$ \\
\hline ET-1 $^{\mathrm{c}}$ & $55.1 \%[31.5-78.6]$ & $41.1 \%[14.2-100.0]$ & $p=0.739$ \\
\hline
\end{tabular}

Abbreviations: DM, diabetes mellitus; ET-1, endothelin-1; HC, hypercholesterolemia; RCT, randomized controlled trial; SNAP, S-nitroso-N-acetyl-D,Lpenicillamine.

Note: Data are presented as median and interquartile range.

${ }^{\mathrm{a}}$ Mann-Whitney $\mathrm{U}$ test between $\mathrm{DM}+\mathrm{HC}$ and controls.

b\% Vasodilation.

c\% Vasoconstriction. 
A

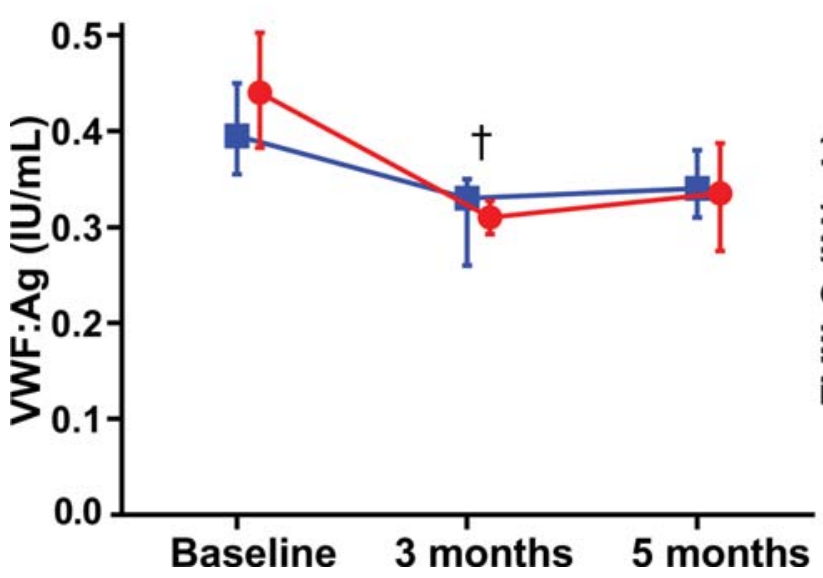

B

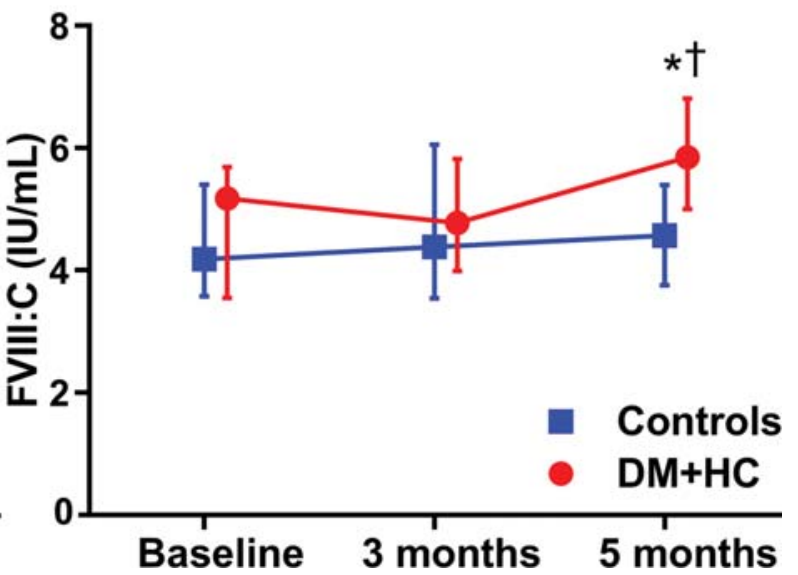

Fig. 2 VWF antigen did not increase during 5 months of follow-up (A), whereas FVIII activity did increase (B). Data presented as mean and 95\% confidence interval. ${ }^{*} p<0.05$ between $\mathrm{DM}+\mathrm{HC}$ and controls. ${ }^{\dagger} p<0.05$ compared with baseline levels in DM $+\mathrm{HC}$ swine. DM, diabetes mellitus; FVIII, factor VIII; HC, hypercholesterolemia; VWF, von Willebrand factor.
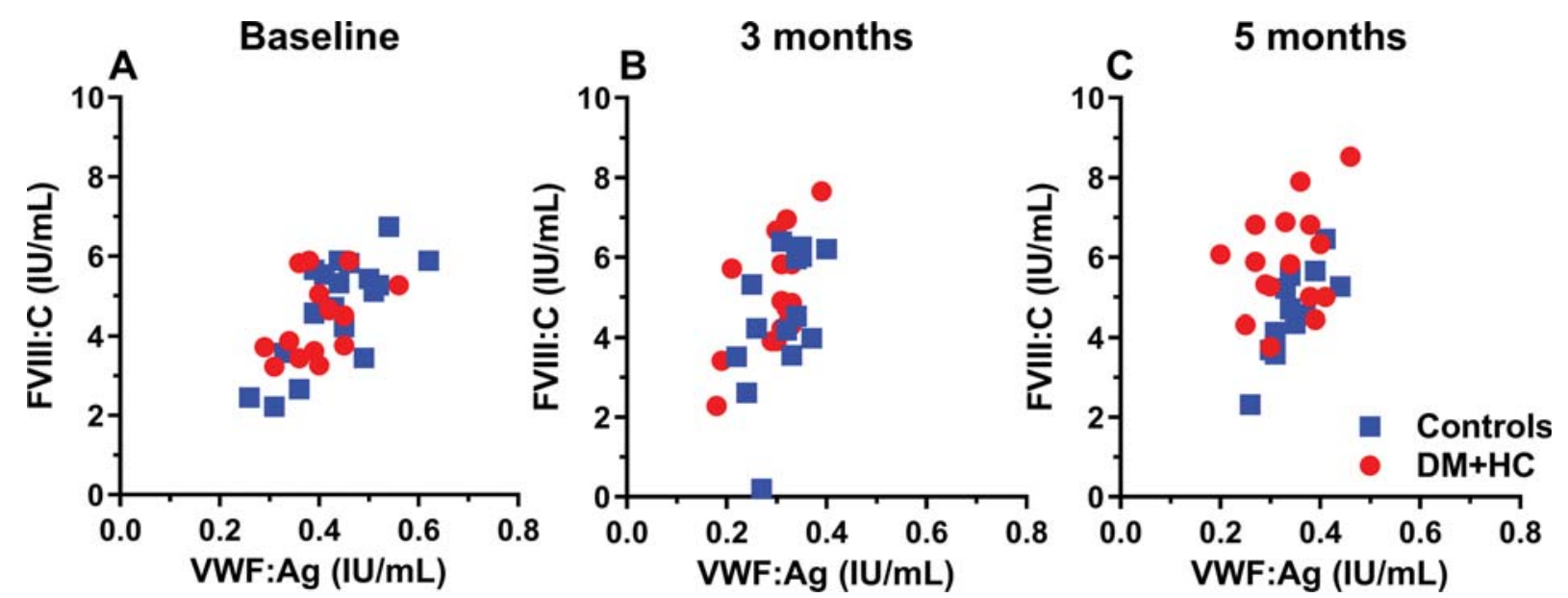

Fig. 3 The correlation between VWF and FVIII disappeared in DM + HC swine during 5 months of follow-up. (A) At baseline, the correlation between VWF:Ag and FVIII:C was $r=0.63(p=0.006)$ in DM $+\mathrm{HC}$ swine and $r=0.44(p=0.116)$ in controls. (B) At 3 months of follow-up, this correlation was $r=0.55(p=0.027)$ in DM $+\mathrm{HC}$ swine and $r=52(0.058)$ in controls. (C) At 5 months, this correlation disappeared in DM $+\mathrm{HC}$ swine $(r=0.18, p=0.513)$, whereas it was strongly present in controls $(r=0.84, p<0.001)$. Outcomes of Spearman's correlation analysis are provided. Ag, antigen; DM diabetes mellitus; FVIII, factor VIII; HC hypercholesterolemia; VWF, von Willebrand factor.
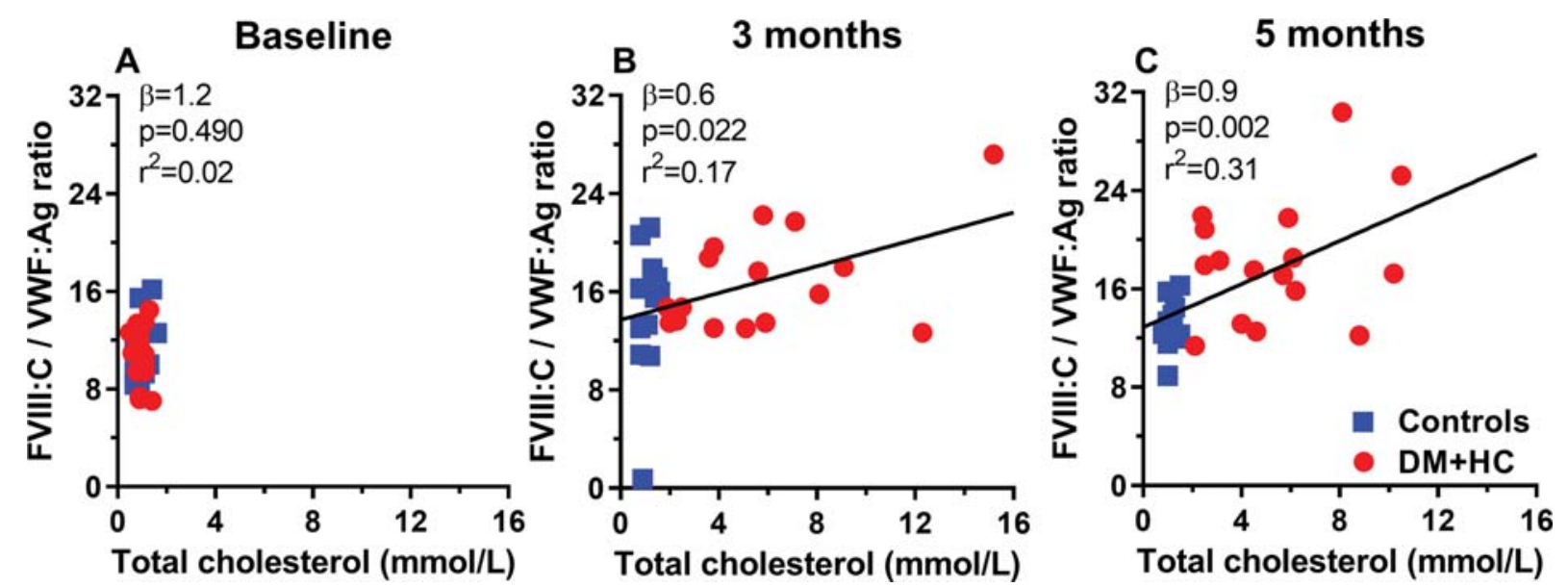

Fig. 4 Higher cholesterol was associated with a higher FVIII/VWF ratio. (A) At baseline, there was no correlation between total cholesterol and FVIII:C/VWF:Ag ratio. (B) At 3 months total cholesterol was correlated with FVIII:C/VWF:Ag, and (C) at 5 months this correlation was even stronger. Outcomes of linear regression analysis are provided. Ag, antigen; DM diabetes mellitus; FVIII, factor VIII; HC hypercholesterolemia; VWF, von Willebrand factor. 
linear association between cholesterol and the FVIII:C/VWF:Ag ratio was $\beta=1.2(p=0.490)$ at baseline, $\beta=0.6(p=0.022)$ at 3 months, and $\beta=0.9(p=0.002)$ at 5 months. Similar associations were found between LDL, HDL, triglycerides, and FVIII:C/VWF:Ag ratio (-Supplementary Fig. S1, available in the online version). Cholesterol was not significantly associated with FVIII:C alone at baseline, 3 months, and 5 months (-Supplementary Fig. S2, available in the online version). Furthermore, higher glucose at 5 months was also associated with higher FVIII:C $(\beta=0.08, p=0.043)$, but not with VWF:Ag ( $\beta$ $=0.00, p=0.120$, - Supplementary Fig. $\mathbf{S 3}$, available in the online version). Together these results indicate that increased plasma levels of cholesterol and glucose result in higher FVIII:C without change of VWF:Ag.

\section{Long-Term Longitudinal Cohort}

In the long-term longitudinal cohort, VWF:Ag was first measured 9 months after induction of $\mathrm{HC}$ and $\mathrm{DM}+\mathrm{HC}$. At that time, both groups had robust dyslipidemia as evidenced by-comparable to the short-term cohort-high levels of cholesterol and triglycerides, whereas fasting hyperglycemia was only present in DM + HC swine (-Table 3 ). Plasma lipid and glucose levels of these swine remained constant between 9 and 15 months of follow-up (-Table 3).

In contrast to the short-term cohort, coronary angiograms showed that from 9 months on, atherosclerosis progressively

Table 3 Characteristics of swine in the long-term longitudinal cohort

\begin{tabular}{|c|c|c|}
\hline & $\mathrm{HC}(n=5)$ & $\mathrm{DM}+\mathrm{HC}(n=5)$ \\
\hline \multicolumn{3}{|c|}{ Triglycerides (mmol/L) } \\
\hline $9 \mathrm{mo}$ & $0.37[0.16-1.33]$ & $0.87[0.39-1.63]$ \\
\hline $12 \mathrm{mo}$ & $0.32[0.23-0.62]$ & $0.41[0.30-1.41]$ \\
\hline $15 \mathrm{mo}$ & $0.42[0.32-1.08]$ & $0.64[0.38-2.46]$ \\
\hline \multicolumn{3}{|c|}{ Total cholesterol (mmol/L) } \\
\hline 9 mo & $18.0[14.1-27.1]$ & $16.1[15.2-26.1]$ \\
\hline $12 \mathrm{mo}$ & $18.6[13.8-25.8]$ & 18.2 [12.6-21.9] \\
\hline $15 \mathrm{mo}$ & $19.0[12.0-23.8]$ & 17.9 [12.9-20.3] \\
\hline \multicolumn{3}{|c|}{ HDL cholesterol (mmol/L) } \\
\hline $9 \mathrm{mo}$ & $5.87[5.44-6.50]$ & $5.02[4.47-6.01]$ \\
\hline $12 \mathrm{mo}$ & $6.10[5.21-6.57]$ & $5.99[4.48-6.52]$ \\
\hline $15 \mathrm{mo}$ & $5.72[5.04-6.36]$ & $5.30[4.33-5.82]$ \\
\hline \multicolumn{3}{|c|}{ LDL cholesterol (mmol/L) } \\
\hline $9 \mathrm{mo}$ & 15.3 [11.6-21.9] & $12.3[11.5-22.4]$ \\
\hline $12 \mathrm{mo}$ & $14.9[11.3-22.2]$ & $15.0[9.4-17.2]$ \\
\hline $15 \mathrm{mo}$ & $15.2[9.4-20.2]$ & $14.0[9.8-17.3]$ \\
\hline \multicolumn{3}{|c|}{ Glucose (mmol/L) } \\
\hline $9 \mathrm{mo}$ & $3.7[3.4-4.8]$ & $11.1[9.1-24.4]$ \\
\hline $12 \mathrm{mo}$ & $4.0[3.8-4.4]$ & $9.4[8.5-20.9]$ \\
\hline $15 \mathrm{mo}$ & $4.8[4.5-5.9]$ & $15.8[9.0-27.3]$ \\
\hline
\end{tabular}

Abbreviations: DM, diabetes mellitus; HC, hypercholesterolemia; HDL, high-density lipoprotein; LDL, low-density lipoprotein.

Note: Data are presented as median and interquartile range. developed in large coronary arteries of both $\mathrm{HC}$ and DM $+\mathrm{HC}$ swine (-Fig. 5A). Histological studies at 15 months also showed clear atherosclerosis in large coronary arteries of both $\mathrm{HC}$ and $\mathrm{DM}+\mathrm{HC}$ swine (-Fig. 5B). Likewise, at 15 months both $\mathrm{HC}$ and $\mathrm{DM}+\mathrm{HC}$ swine had endothelial dysfunction as evidenced by significantly increased vasoconstrictor responses to ET-1 ( - Table 4), which was, at least partly, due to a loss of endothelium-dependent endothelin receptor B-mediated dilation as discussed before. ${ }^{18}$

\section{Increase of VWF:Ag during 15 Months of Follow-Up}

In line with the progressive development of atherosclerosis, VWF levels gradually increased over time in $\mathrm{HC}$ and DM $+\mathrm{HC}$ swine (-Fig. 6). In HC swine, VWF:Ag was $0.27 \mathrm{IU} / \mathrm{mL}$ [0.23-0.40] at 9 months, $0.34 \mathrm{IU} / \mathrm{mL}$ [0.28-0.36] at 12 months, and $0.37 \mathrm{IU} / \mathrm{mL}$ [0.32-0.42] at 15 months (-Fig. 6). Thus, VWF:Ag was significantly higher at 15 months compared with 9 months $(p=0.042)$ and 12 months ( $p=0.043$, - Fig. 6). In DM + HC swine, VWF:Ag was $0.25 \mathrm{IU} / \mathrm{mL}[0.24-0.33]$ at 9 months and significantly increased to $0.30 \mathrm{IU} / \mathrm{mL}[0.28-0.35](p=0.042)$ at 12 months and $0.33 \mathrm{IU} / \mathrm{mL}$ [0.32-0.37] $(p=0.042)$ at 15 months (-Fig. 6).

\section{Discussion}

In the present study in swine exposed to cardiovascular risk factors, we found that FVIII levels, but not VWF levels, increased after 5 months of exposure to DM and HC. This increase in FVIII levels was independent of VWF levels, which remained stable over time, and was associated with plasma cholesterol, LDL, HDL, triglycerides, and glucose levels. Five months of exposure to HC and DM did not lead to endothelial dysfunction or atherosclerosis. However, long-term (15 months) exposure to DM and HC did result in increased VWF levels, at a time when endothelial dysfunction and atherosclerosis were present.

These results suggest that endothelial dysfunction and atherosclerosis are the major determinants of the increase in VWF levels over time in swine with DM and HC. Similarly, a study in mice found that DM and hyperglycemia eventually led to increased VWF levels via endothelial dysfunction. ${ }^{24}$ Our findings may explain the results of previous studies in humans showing an association between high VWF levels and extensive atherosclerosis, cardiovascular disease, and worse cardiovascular outcomes. ${ }^{5,6}$ Since atherosclerosis and endothelial dysfunction lead to both higher VWF levels and worse cardiovascular outcomes, VWF may be seen as a biomarker for advanced cardiovascular disease. In line with this, two large population-based cohort studies concluded that VWF is primarily a biomarker of cardiovascular disease, instead of a strong causal factor. ${ }^{25,26}$ However, VWF may, by its procoagulant function promoting platelet aggregation and thrombosis, also have an additional direct effect on cardiovascular outcomes. $^{14,27}$ Therefore, well-designed studies are needed to investigate the contribution of each of these two mechanisms (i.e., indirect role of VWF as biomarker vs. direct procoagulant role of VWF) in the 


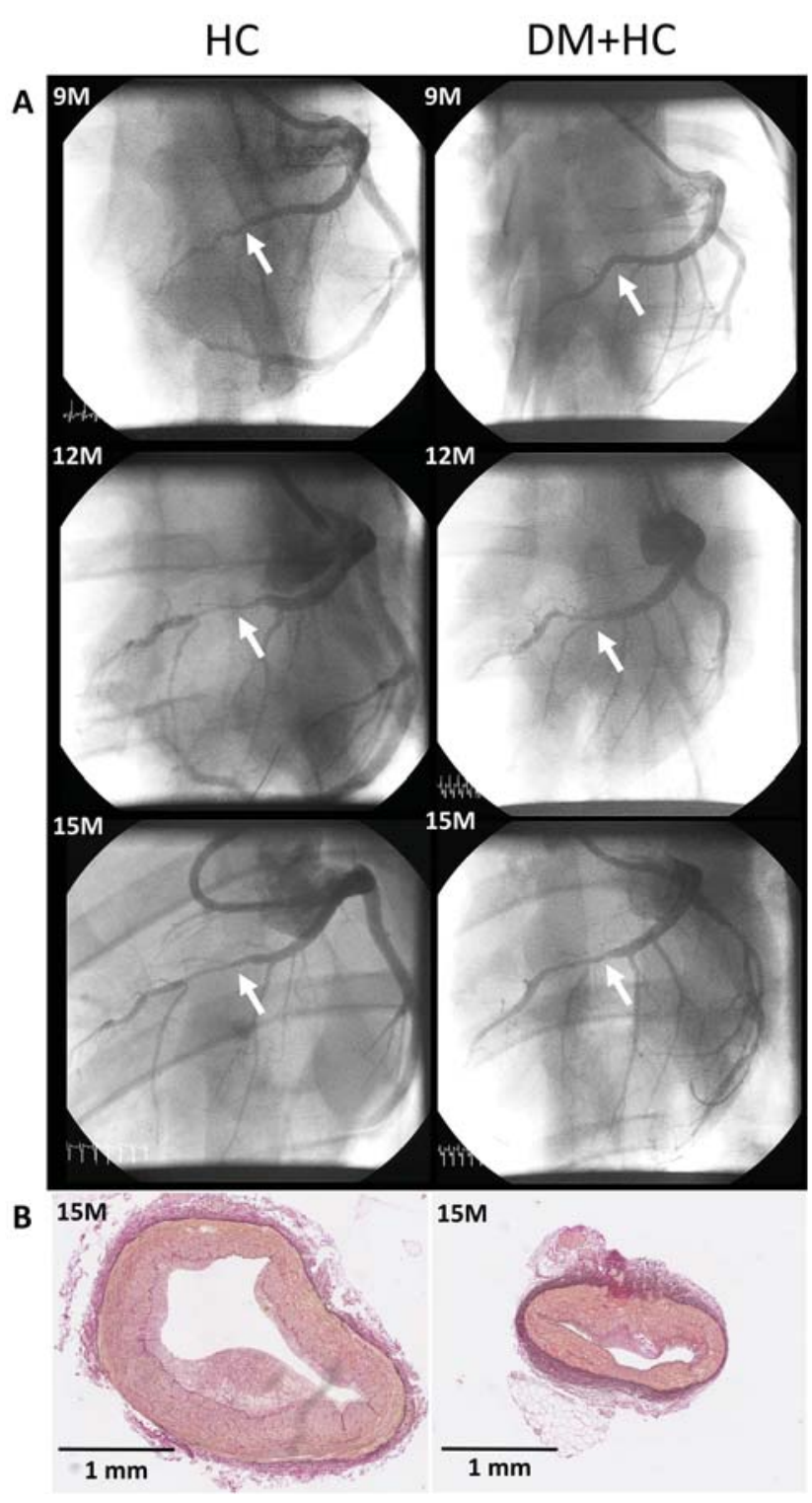

Fig. 5 There were clear signs of atherosclerosis present in large coronary arteries of both $\mathrm{HC}$ and $\mathrm{DM}+\mathrm{HC}$ swine after 15 months of follow-up in the long-term longitudinal cohort. (A) Examples of progressive, from $9(9 \mathrm{M}), 12(12 \mathrm{M})$, and $15(15 \mathrm{M})$ months, coronary lesions on coronary angiograms at a left anterior oblique $30^{\circ}$ and a cranial $0^{\circ}$ view of both $\mathrm{HC}$ and $\mathrm{DM}+\mathrm{HC}$ swine. (B) Typical examples resorcin-fuchsin stained large coronary arteries of atherosclerosis of $\mathrm{HC}$ and $\mathrm{DM}+\mathrm{HC}$ swine at 15 months $(15 \mathrm{M})$ of the long-term longitudinal cohort. DM diabetes mellitus; HC hypercholesterolemia.

relation between VWF and cardiovascular disease and outcomes. Such information may provide more insights into how effective potential VWF-targeted treatment for prevention or treatment of cardiovascular disease may be.

Endothelial dysfunction and atherosclerosis may lead to higher VWF levels due to increased VWF release from dysfunctional endothelial cells. ${ }^{28}$ However, atherosclerosis development may also attenuate VWF clearance by macrophages. It is known that macrophages play a critical role in VWF clearance, by binding and clearing VWF in a dosedependent manner. ${ }^{29,30}$ At the same time, macrophages play a key role in development of atherosclerosis. ${ }^{31}$ Internal-

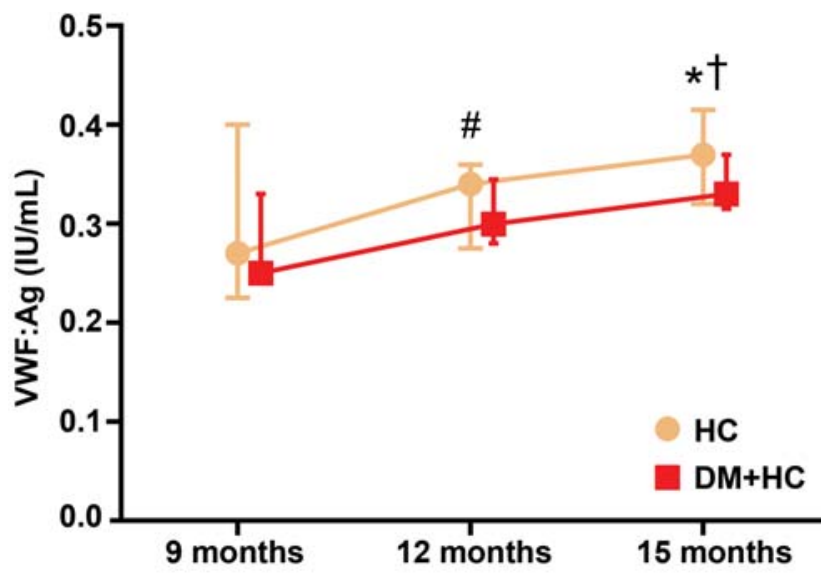

Fig. 6 VWF levels progressively increased in $\mathrm{HC}$ and $\mathrm{DM}+\mathrm{HC}$ swine in the long-term longitudinal cohort. Data presented as mean and 95\% confidence interval. * $p<0.05$ compared with baseline levels in both groups. ${ }^{\dagger} p<0.05$ compared with levels at 12 months in $\mathrm{HC}$ swine. ${ }^{\#} p<0.05$ compared with baseline levels in DM + HC swine. DM diabetes mellitus; HC hypercholesterolemia; VWF, von Willebrand factor.

ization of LDL particles by macrophages and formation of foam cells can be accompanied by macrophage apoptosis. ${ }^{31}$ Therefore, it is very likely that changes in macrophages during the development of atherosclerosis may contribute to the increase of VWF levels over time. In line with this, it has been shown that diet-induced obesity leads to higher VWF and FVIII levels in mice via both increased synthesis and reduced clearance of $\mathrm{VWF}^{32}$

Interestingly, FVIII levels increased shortly after induction of HC and hyperglycemia, whereas VWF levels remained unchanged, resulting in the disappearance of the correlation between VWF and FVIII levels in DM + HC swine after 5 months of follow-up. In line with this, a recent large study in the general population of 5,778 individuals found that higher fasting glucose was associated with higher FVIII levels, even after adjustment for confounders, whereas a RCT in 12 healthy volunteers found that hyperglycemia did not affect VWF levels during a short-term follow-up period. ${ }^{33,34}$ On the long term, it has also been shown that patients with DM have higher VWF and FVIII levels than healthy individuals in the general population, possibly due to long-term consequences of DM. ${ }^{35-37}$ However, in contrast, the Biobreeding Wistar rat, which is a model of spontaneous autoimmune type $1 \mathrm{DM}$, showed similar VWF levels compared with controls during a long-term follow-up period. ${ }^{38}$ There are several possible mechanisms by which hyperglycemia may increase FVIII levels. Hyperglycemia may lead to loss of endothelial glycocalyx and subsequently activation of the coagulation system, resulting in a direct increase of FVIII levels. ${ }^{39}$ Additionally, increased oxidative stress during hyperglycemia may lead to higher FVIII levels. ${ }^{33}$ Furthermore, FVIII levels may be directly increased due to $\mathrm{HC}$, as the region within the $\mathrm{C} 2$ domain of FVIII involved in VWF binding can also bind lipids. ${ }^{40}$ Therefore, in severe HC, FVIII may (besides VWF) bind lipids, thereby prolonging the half-life of FVIII, independent of VWF. In line with this, we found that a higher FVIII/VWF ratio 
Table 4 Endothelial dysfunction in coronaries of $\mathrm{HC}$ and $\mathrm{DM}+\mathrm{HC}$ in the long term cohort

\begin{tabular}{|l|l|l|l|l|}
\hline & HC $(\boldsymbol{n}=\mathbf{4})$ & DM + HC $(\boldsymbol{n}=\mathbf{5})$ & Controls $(\boldsymbol{n}=\mathbf{6 - 1 8})$ & $p$-Value ${ }^{\mathbf{b}}$ \\
\hline Bradykinin $^{\mathrm{c}}$ & $82.3 \%[74.6-87.9]$ & $89.2 \%[78.6-90.7]$ & $77.0 \%[62.7-88.4]$ & $p=0.400$ \\
\hline SNAP $^{\mathrm{C}}$ & $57.2 \%[48.8-63.2]$ & $40.7 \%[33.6-66.0]$ & $24.8 \%[17.8-48.9]$ & $p=0.321$ \\
\hline ET- $^{\text {d }}$ & $63.4 \%[58.2-80.9]$ & $58.8 \%[46.5-82.9]$ & $19.7 \%[9.1-28.4]$ & $p=0.003$ \\
\hline
\end{tabular}

Abbreviations: DM, diabetes mellitus; ET-1, endothelin 1; HC, hypercholesterolemia; SNAP, S-nitroso-N-acetyl-D,L-penicillamine.

Note: Data are presented as median and interquartile range.

${ }^{a}$ Controls: bradykinin $n=6$, SNAP $n=18$, ET- $n=11$.

${ }^{\mathrm{b}}$ Kruskal-Wallis test between all three groups.

c\% Vasodilation.

d\% Vasoconstriction.

was associated with higher levels of total cholesterol, LDL, HDL, and triglycerides. Also, it has previously been shown in mice that FVIII-lipid binding, besides VWF-FVIII binding, synergistically prolongs the plasma survival of FVIII. ${ }^{41}$ During normal conditions the role of VWF-FVIII binding on FVIII survival is larger, because of the high binding affinity of VWF with FVIII $(0.5 \mathrm{nM}) .{ }^{42,43}$ However, in a high lipid environment, lipids may, despite a lower binding affinity to FVIII ( $6 \mathrm{nM}$, compared with VWF), contribute significantly to FVIII survival by additional lipid-FVIII binding. ${ }^{41,42}$ In line with this, we have found that cholesterol is associated with the FVIII:C/VWF:Ag ratio stronger than that with the FVIII: $\mathrm{C}$ ratio alone.

The current study may also have important implications for patients with von Willebrand disease (VWD), which is caused by reduced VWF levels, leading to bleeding symptoms. ${ }^{44-47}$ Our results suggest that endothelial dysfunction and atherosclerosis mediate comorbidity-dependent increase in VWF levels in patients with type 1 VWD. ${ }^{10}$ Similarly, our data are in accordance with human data showing that body mass index, which is highly related to endothelial dysfunction and atherosclerosis, was associated with higher VWF and FVIII levels in type 1 VWD patients. ${ }^{48}$ Altogether, these data suggest that not only the incidence of comorbidities, but also the severity of endothelial dysfunction and atherosclerosis modulates VWF and FVIII levels. ${ }^{12,31,48,49}$ Prospective studies in VWD patients are needed to investigate the effect of the comorbidity-related increase of VWF and FVIII levels on the bleeding tendency of these patients. ${ }^{50}$

To our knowledge, this is the first study to investigate the pathophysiological mechanisms of the increase of VWF and FVIII levels in a relevant large animal model with cardiovascular risk factors using a RCT design. For this study, we employed swine, which have proven to be an excellent model for human metabolic syndrome and cardiovascular disease, mainly due to their similarities to humans with regards to their glucose and lipid metabolism, cardiovascular anatomy, physiology, and myocardial metabolism. ${ }^{51-53}$ Swine also show great resemblance to humans with regards to both VWF and VWD. ${ }^{54}$ In this study, we showed that hyperglycemia and HC increase FVIII levels, independent of VWF. In addition, we showed that VWF levels only increased when endothelial dysfunction and atherosclerosis were present. Nevertheless, this study has several potential limitations. First, the most important limitation is that we did not measure baseline VWF levels in the long-term longitudinal cohort study, as no citrated blood was collected at inclusion in the study. Notwithstanding this limitation, we found that VWF levels progressively increased in $\mathrm{HC}$ and $\mathrm{DM}+\mathrm{HC}$ swine with overt endothelial dysfunction and atherosclerosis during the period between 9- and 15- months of follow-up. Second, there was no control group in the long-term cohort study. Finally, we did not measure FVIII levels in the longterm longitudinal cohort, as citrate plasma was not available anymore to measure FVIII levels. However, we clearly show in the short-term RCT cohort that FVIII levels increased because of hyperglycemia and HC, independent of VWF.

In conclusion, the present study shows that endothelial dysfunction and atherosclerosis lead to an increase of VWF levels in swine, whereas hyperglycemia and HC already increased FVIII levels prior to development of endothelial dysfunction and atherosclerosis (-Fig. 7). Therefore, VWF seems to be a biomarker for advanced cardiovascular disease. These findings contribute to a better understanding of the association between high VWF and FVIII levels and atherosclerosis, cardiovascular disease, and a worse cardiovascular outcome in humans.

\section{What is known about this topic?}

- VWF and FVIII levels are associated with an increased risk of cardiovascular disease.

- VWF and FVIII levels increase with aging, possibly due to incidence of comorbidities at an older age.

\section{What does this paper add?}

- VWF increase over time is mediated via endothelial dysfunction and atherosclerosis, whereas short-term hyperglycemia and dyslipidemia increase FVIII.

- VWF seems to be a biomarker for advanced cardiovascular disease, explaining its association with cardiovascular disease and outcomes.

\section{Authors' Contributions}

F.A. and J.v.d.W. designed the study, collected the data, performed the statistical analysis, interpreted the data, and wrote the manuscript. O.S., I.H., M.P.M.d.M., D.M., D.J. 


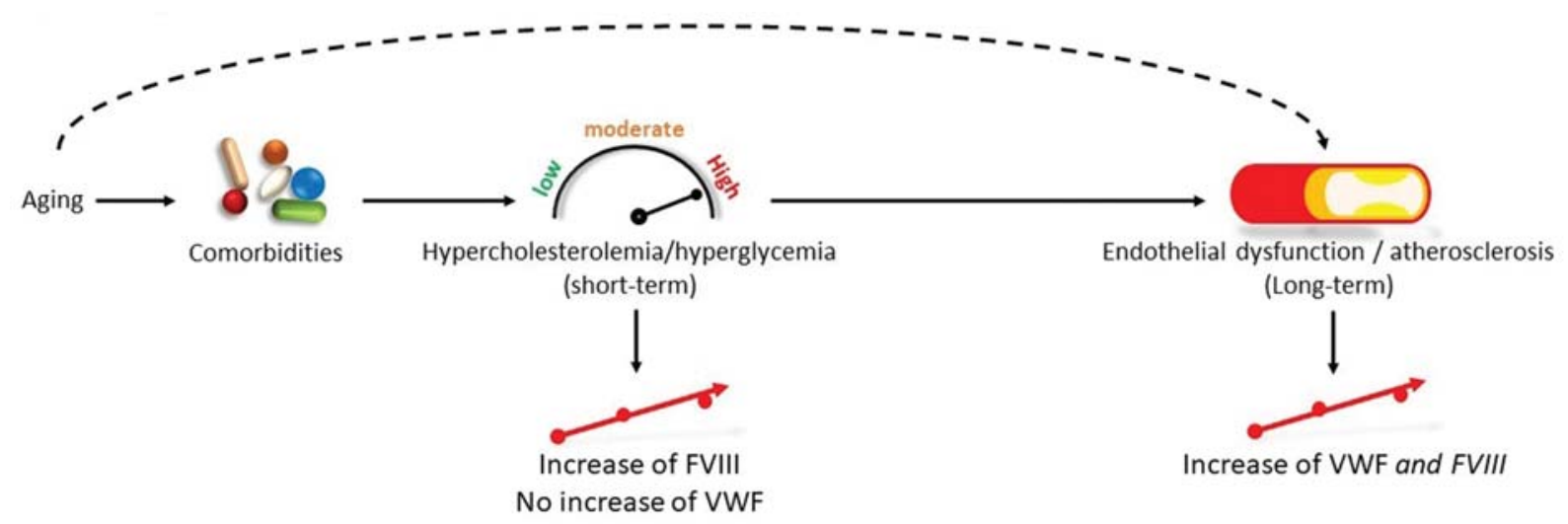

Fig. 7 Summary of the association between comorbidities, endothelial dysfunction, and atherosclerosis and VWF and FVIII levels. Aging gives a higher chance for comorbidities, which leads to an increase of FVIII via HC and hyperglycemia, but does not influence VWF. Over a long time period, comorbidities lead to endothelial dysfunction and atherosclerosis, which increase VWF. Because of the strong physiologic association between VWF and FVIII, an increase of VWF will subsequently also lead to higher FVIII levels. DM diabetes mellitus; FVIII, factor VIII; HC hypercholesterolemia; VWF, von Willebrand factor.

D., and F.W.G.L. designed the study, collected the data, interpreted the data, and critically revised the manuscript. All authors gave their consent to the final version of the manuscript.

\section{Data Sharing Statement}

Original data can be obtained by sending an e-mail to the corresponding author (f.leebeek@erasmusmc.nl).

\section{Funding}

This study was supported by grants from the European Commission FP7-Health-2010 grant MEDIA-261409, the German Center for Cardiovascular Research (DZHK; $81 Z 0600207$ to D.M.), the Netherlands CardioVascular Research Initiative: an initiative with support of the Dutch Heart Foundation [CVON2014-11 (RECONNECT), CVON2016-ARENA-Prime], and the Academy of Finland 251272, Finnish Diabetes Research Foundation, and Finnish Foundation for Cardiovascular Research.

\section{Conflict of Interest}

F.A. received the CSL Behring-Professor Heimburger Award 2018 and a travel grant from Sobi. F.W.G.L. received research support from CSL Behring and Shire/Takeda for performing the WiN study; is a consultant for uniQure, Novo Nordisk, and Shire/Takeda, the fees of which go to the institution; and has received a travel grant from Sobi. He is also a DSMB member for a study by Roche.

\section{Acknowledgments}

The authors thank Ilona Krabbendam, Richard van Duin, and Ruben van Drie (Erasmus MC, Rotterdam, The Netherlands) for their expert technical support, and the analysts of the Erasmus MC Hemostasis Laboratory for performing the VWF:Ag and FVIII:C measurements.

\section{References}

1 Leebeek FW, Eikenboom JC. von Willebrand's disease. N Engl J Med 2016;375(21):2067-2080
2 Springer TA. von Willebrand factor, Jedi knight of the bloodstream. Blood 2014;124(09):1412-1425

3 Sonneveld MA, de Maat MP, Leebeek FW. Von Willebrand factor and ADAMTS13 in arterial thrombosis: a systematic review and meta-analysis. Blood Rev 2014;28(04):167-178

4 Sonneveld MA, Franco OH, Ikram MA, et al. Von Willebrand factor, ADAMTS13, and the risk of mortality: the Rotterdam study. Arterioscler Thromb Vasc Biol 2016;36(12):2446-2451

5 Sonneveld MA, Cheng JM, Oemrawsingh RM, et al. Von Willebrand factor in relation to coronary plaque characteristics and cardiovascular outcome. Results of the ATHEROREMO-IVUS study. Thromb Haemost 2015;113(03):577-584

6 Sonneveld MA, van Dijk AC, van den Herik EG, et al. Relationship of Von Willebrand factor with carotid artery and aortic arch calcification in ischemic stroke patients. Atherosclerosis 2013;230(02): 210-215

7 Kovacevic KD, Jilma B, Zhu S, et al. von Willebrand factor predicts mortality in ACS patients treated with potent P2Y12 antagonists and is inhibited by aptamer BT200 ex vivo. Thromb Haemost 2020;120(09):1282-1290

8 Rydz N, Grabell J, Lillicrap D, James PD. Changes in von Willebrand factor level and von Willebrand activity with age in type 1 von Willebrand disease. Haemophilia 2015;21(05): 636-641

9 Sanders YV, Giezenaar MA, Laros-van Gorkom BA, et al;WiN study group. von Willebrand disease and aging: an evolving phenotype. J Thromb Haemost 2014;12(07):1066-1075

10 Atiq F, Meijer K, Eikenboom J, et al;WiN study group. Comorbidities associated with higher von Willebrand factor (VWF) levels may explain the age-related increase of VWF in von Willebrand disease. Br J Haematol 2018;182(01):93-105

11 Abou-Ismail MY, Ogunbayo GO, Secic M, Kouides PA. Outgrowing the laboratory diagnosis of type 1 von Willebrand disease: a two decade study. Am J Hematol 2018;93(02):232-237

12 Seaman CD, Ragni MV. The effect of age on von Willebrand factor and bleeding symptoms in von Willebrand disease. Thromb Haemost 2020;120(08):1159-1165

13 Lip GY, Blann A. von Willebrand factor: a marker of endothelial dysfunction in vascular disorders? Cardiovasc Res 1997;34(02): 255-265

14 Montoro-García S, Shantsila E, Lip GY. Potential value of targeting von Willebrand factor in atherosclerotic cardiovascular disease. Expert Opin Ther Targets 2014;18(01):43-53

15 Buchtele N, Schwameis M, Gilbert JC, Schörgenhofer C, Jilma B. Targeting von Willebrand factor in ischaemic stroke: focus on clinical evidence. Thromb Haemost 2018;118(06):959-978 
16 van den Dorpel MMP, Heinonen I, Snelder SM, et al. Early detection of left ventricular diastolic dysfunction using conventional and speckle tracking echocardiography in a large animal model of metabolic dysfunction. Int J Cardiovasc Imaging 2018;34(05): 743-749

17 Münster AM, Olsen AK, Bladbjerg EM. Usefulness of human coagulation and fibrinolysis assays in domestic pigs. Comp Med 2002;52(01):39-43

18 Sorop O, van den Heuvel M, van Ditzhuijzen NS, et al. Coronary microvascular dysfunction after long-term diabetes and hypercholesterolemia. Am J Physiol Heart Circ Physiol 2016;311(06): H1339-H1351

19 van den Heuvel M, Sorop O, Koopmans SJ, et al. Coronary microvascular dysfunction in a porcine model of early atherosclerosis and diabetes. Am J Physiol Heart Circ Physiol 2012;302(01): H85-H94

20 van de Wouw J, Sorop O, van Drie RWA, et al. Perturbations in myocardial perfusion and oxygen balance in swine with multiple risk factors: a novel model of ischemia and no obstructive coronary artery disease. Basic Res Cardiol 2020;115(02):21

21 Sorop O, Heinonen I, van Kranenburg M, et al. Multiple common comorbidities produce left ventricular diastolic dysfunction associated with coronary microvascular dysfunction, oxidative stress, and myocardial stiffening. Cardiovasc Res 2018;114(07):954-964

22 Borbouse L, Dick GM, Payne GA, et al. Contribution of BK(Ca) channels to local metabolic coronary vasodilation: effects of metabolic syndrome. Am J Physiol Heart Circ Physiol 2010;298 (03):H966-H973

23 Hasdai D, Mathew V, Schwartz RS, et al. Enhanced endothelin-Breceptor-mediated vasoconstriction of small porcine coronary arteries in diet-induced hypercholesterolemia. Arterioscler Thromb Vasc Biol 1997;17(11):2737-2743

24 Xiang Y, Cheng J, Wang D, et al. Hyperglycemia repression of miR24 coordinately upregulates endothelial cell expression and secretion of von Willebrand factor. Blood 2015;125(22):3377-3387

25 Van Schie MC, Wieberdink RG, Koudstaal PJ, et al. Genetic determinants of von Willebrand factor plasma levels and the risk of stroke: the Rotterdam Study. J Thromb Haemost 2012;10 (04):550-556

26 van Loon JE, Kavousi M, Leebeek FW, et al. von Willebrand factor plasma levels, genetic variations and coronary heart disease in an older population. J Thromb Haemost 2012;10(07):1262-1269

27 Shahidi M. Thrombosis and von Willebrand factor. Adv Exp Med Biol 2017;906:285-306

28 Lenting PJ, Christophe OD, Denis CV. von Willebrand factor biosynthesis, secretion, and clearance: connecting the far ends. Blood 2015;125(13):2019-2028

29 O'Sullivan JM, Ward S, Lavin M, O'Donnell JS. von Willebrand factor clearance - biological mechanisms and clinical significance. Br J Haematol 2018;183(02):185-195

30 Ward SE, O'Sullivan JM, Drakeford C, et al. A novel role for the macrophage galactose-type lectin receptor in mediating von Willebrand factor clearance. Blood 2018;131(08):911-916

31 Rocha VZ, Libby P. Obesity, inflammation, and atherosclerosis. Nat Rev Cardiol 2009;6(06):399-409

32 Michels A, Dwyer CN, Mewburn J, et al. von Willebrand factor is a critical mediator of deep vein thrombosis in a mouse model of diet-induced obesity. Arterioscler Thromb Vasc Biol 2020;40(12): 2860-2874

33 van der Toorn FA, de Mutsert R, Lijfering WM, Rosendaal FR, van Hylckama Vlieg A. Glucose metabolism affects coagulation factors: the NEO study. J Thromb Haemost 2019;17(11):1886-1897

34 Kotzailias N, Graninger M, Knechtelsdorfer M, Jilma B. Acute effects of hyperglycaemia on plasma concentration of soluble Pselectin and von Willebrand factor in healthy volunteers -a prospective randomised double blind controlled study. Thromb Res 2009;123(03):452-459

35 Conlan MG, Folsom AR, Finch A, et al. Associations of factor VIII and von Willebrand factor with age, race, sex, and risk factors for atherosclerosis. The Atherosclerosis Risk in Communities (ARIC) Study. Thromb Haemost 1993;70(03):380-385

36 Kessler L, Wiesel ML, Attali P, Mossard JM, Cazenave JP, Pinget M. Von Willebrand factor in diabetic angiopathy. Diabetes Metab 1998;24(04):327-336

37 Seligman BG, Biolo A, Polanczyk CA, Gross JL, Clausell N. Increased plasma levels of endothelin 1 and von Willebrand factor in patients with type 2 diabetes and dyslipidemia. Diabetes Care 2000;23(09):1395-1400

38 Ribau JC, Hatton MW, Richardson M. Changes in the aortic endothelium and plasma von Willebrand factor levels during the onset and progression of insulin-dependent diabetes in BB rats. Atherosclerosis 1998;139(02):291-299

39 Nieuwdorp M, van Haeften TW, Gouverneur MC, et al. Loss of endothelial glycocalyx during acute hyperglycemia coincides with endothelial dysfunction and coagulation activation in vivo. Diabetes 2006;55(02):480-486

40 Peng A, Straubinger RM, Balu-Iyer SV. Phosphatidylinositol containing lipidic particles reduces immunogenicity and catabolism of factor VIII in hemophilia a mice. AAPS J 2010;12(03):473-481

41 Pisal DS, Balu-lyer SV. Phospholipid binding improves plasma survival of factor VIII. Thromb Haemost 2010;104(05):1073-1075

42 Saenko E, Sarafanov A, Greco N, et al. Use of surface plasmon resonance for studies of protein-protein and protein-phospholipid membrane interactions. Application to the binding of factor VIII to von Willebrand factor and to phosphatidylserine-containing membranes. J Chromatogr A 1999;852(01):59-71

43 Gilbert GE, Kaufman RJ, Arena AA, Miao H, Pipe SW. Four hydrophobic amino acids of the factor VIII C2 domain are constituents of both the membrane-binding and von Willebrand factor-binding motifs. J Biol Chem 2002;277(08):6374-6381

44 Leebeek FWG, Atiq F. How I manage severe von Willebrand disease. Br J Haematol 2019;187(04):418-430

45 Atiq F, Schütte LM, Looijen AEM, et al. von Willebrand factor and factor VIII levels after desmopressin are associated with bleeding phenotype in type 1 VWD. Blood Adv 2019;3(24):4147-4154

46 Sharma R, Flood VH. Advances in the diagnosis and treatment of Von Willebrand disease. Blood 2017;130(22):2386-2391

47 Sadler JE. Von Willebrand disease type 1: a diagnosis in search of a disease. Blood 2003;101(06):2089-2093

48 Atiq F, Fijnvandraat K, van Galen KPM, et al;WiN study group. BMI is an important determinant of VWF and FVIII levels and bleeding phenotype in patients with von Willebrand disease. Am J Hematol 2019;94(08):E201-E205

49 Lovren F, Teoh H, Verma S. Obesity and atherosclerosis: mechanistic insights. Can J Cardiol 2015;31(02):177-183

50 Leebeek FWG, Susen S. Von Willebrand disease: clinical conundrums. Haemophilia 2018;24(Suppl 6):37-43

51 Spurlock ME, Gabler NK. The development of porcine models of obesity and the metabolic syndrome. J Nutr 2008;138(02): 397-402

52 Zhang X, Lerman LO. Investigating the metabolic syndrome: contributions of swine models. Toxicol Pathol 2016;44(03): 358-366

53 Sorop O, van de Wouw J, Chandler S, et al. Experimental animal models of coronary microvascular dysfunction. Cardiovasc Res 2020;116(04):756-770

54 Nichols TC, Bellinger DA, Merricks EP, et al. Porcine and canine von Willebrand factor and von Willebrand disease: hemostasis, thrombosis, and atherosclerosis studies. Thrombosis 2010; 2010:461238 